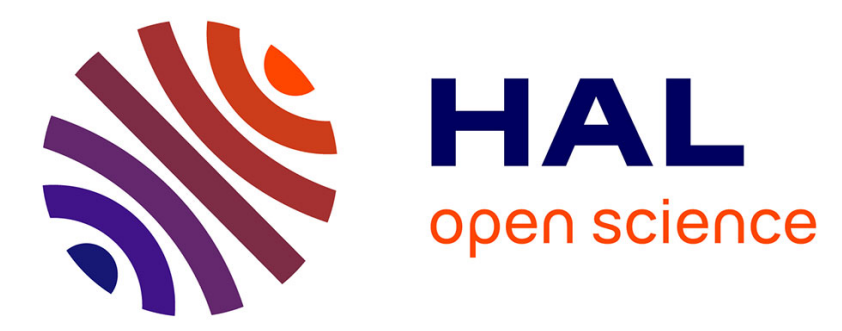

\title{
Mechanical Strength Enhancement by Grain Size Reduction in a Soft Colloidal Polycrystal
}

\author{
Ahmed Mourchid, Imane Boucenna, Florent Carn
}

\section{To cite this version:}

Ahmed Mourchid, Imane Boucenna, Florent Carn. Mechanical Strength Enhancement by Grain Size Reduction in a Soft Colloidal Polycrystal. Soft Matter, 2021. hal-03084855v2

\section{HAL Id: hal-03084855 \\ https://hal.science/hal-03084855v2}

Submitted on 29 Nov 2021

HAL is a multi-disciplinary open access archive for the deposit and dissemination of scientific research documents, whether they are published or not. The documents may come from teaching and research institutions in France or abroad, or from public or private research centers.
L'archive ouverte pluridisciplinaire HAL, est destinée au dépôt et à la diffusion de documents scientifiques de niveau recherche, publiés ou non, émanant des établissements d'enseignement et de recherche français ou étrangers, des laboratoires publics ou privés. 


\title{
Mechanical Strength Enhancement by Grain Size Reduction in a Soft Colloidal Polycrystal
}

\author{
Ahmed Mourchid*, Imane Boucenna, Florent Carn
}

Matière et Systèmes Complexes (MSC), UMR 7057 CNRS and Université de Paris 10 rue Alice Domon et Léonie Duquet, 75205 Paris Cedex 13, France

*ahmed.mourchid@univ-paris-diderot.fr

Keywords: colloids; polycrystals; grain refinement; mechanical properties; Hall-Petch.

\begin{abstract}
It has long been known that the mechanical strength of finely-grained solid state polycrystals could be enhanced when the grain size is reduced. Indeed, the equation linking the yield stress and the inverse square root of grain size was introduced in the 1950s by Hall and Petch. Since then this relationship has been widely used to engineer structural metals and alloys. To date, no similar behavior has been reported in materials other than atomic systems where the grain size usually lies in the nanometric range. The purpose of the present work is to study the influence of grain size on the mechanical strength enhancement of soft colloidal 'alloy' made of micellar polycrystalline grains and silica nanoparticles. The nanoparticles act as nucleation sites and their concentration promotes the variation of the polycrystalline grain size. This system bears resemblance to solid state polycrystals, however the achieved grain length scale is situated in the micrometric domain. We show that the grain size evolves non-monotonically, first decreasing then increasing, when the nanoparticle concentration increases. Our main result is that the yield stress rigorously obey HallPetch law and follows a linear variation as a function of the inverse square root of grain diameter. We believe that our experimental approach offers new possibilities to study poorly understood mechanical aspects of polycrystalline and nanocrystalline structures, such as their plasticity, with non-destructive techniques.
\end{abstract}




\section{Introduction}

Topological defects are well-known to impact many important properties of crystalline materials such as their mechanical behavior [1-6]. One such effect that has been beneficially implemented in applications for decades is strength hardening in solid state metals. Indeed, the formation of grain boundaries and their multiplication by grain refinement lead to peculiar yield stress enhancement which usually shows a 2 to 10 folds increase as compared to the unrefined materials $[1,3,7]$. This well-known relation between yield, flow and cleavage in solid polycrystalline metals, theorized by Hall and Petch in the early 1950 's, is commonly used to engineer polycrystalline solids by refining the size of grains to less than $1 \mu \mathrm{m}$. It predicts that the yield stress follows a linear relation as a function of the inverse square root of grain diameter [8]. The yield stress enhancement is due to the presence of many interfaces, i.e., grain boundaries, which constitute an obstacle to dislocation motion and act as pinning points [8-10]. There is general agreement as to the mechanism behind Hall-Petch law in atomic systems and many studies have shown clear increase in hardness as grain sizes decrease strictly following the established relationship [7]. The strength enhancement usually comes at the expense of poor ductility hampering the effectiveness of micro- and nano-engineered materials and thus limiting their applications. However, deviations from this pathway are being reported as the grain size falls into the nanometric scale. Indeed, in this range, there is agreement that the mechanical properties are furthermore dictated by grain boundary sliding, rotation, or diffusion rather than by dislocation pile-up $[11,12]$. This points again to widened size impact which, in some cases, is at the origin of novel properties of nanomaterials [13-17]. Remarkably, in the nanometric size range, super-plasticity, which gives rise to highly ductile materials, was observed and is believed to be the consequence of adequate tailoring of the microstructure $[14,15]$. Size-dependent softening in small systems was also discovered in nanocrystalline materials [16] and nano-objects [17]. The last findings were observed in nanometric metallic particles subjected to small mechanical solicitations and at relatively low temperature. The observed shape modification was ascribed to surface-diffusion mediated by pseudoelasticity.

All these mechanisms have attracted huge interest owing to the various opportunities they could offer for applications in the light of the large number of small systems that could be both easily designed and studied with newly advanced nanoscopic techniques [18, 19]. Historically, the reports have focused only on solid state metals and their alloys because they constitute an important class of structural materials and are now widely used in nanodevices. However, studying the above 
properties at the nanoscale is a difficult task [13]. It is particularly challenging to probe nanomaterials by using current in-situ techniques which could easily induce sample damage and further complicate data analysis. It is thus barely assured that the observed effects are exempt from technique-induced artefacts at the nanometric size.

An alternative to the solid state materials is provided by soft colloidal materials which are frequently and successfully investigated as model materials to study fundamental physical phenomena ranging from phase transition, structuring in a variety of ordered phases, to glassy dynamics on a notably widened length and time scales [20-24]. Their extensive use is also motivated by the facile means that leads to their synthesis and manipulation, coupled to their direct characterization by several non-destructive techniques [25]. Their relevance over solid state materials is closely related to the fact that their dynamics evolve in large time scales and their structure shows characteristic lengths in a much larger domain, typically at the microscopic level.

Among the recent studies in this field, a new strategy emerged and consisted of adding small amounts of nanoparticles (NPs) to a system of different nature [26-34]. It was successfully shown that when a phase transition is induced in such a system, microscopic segregation occurs and results in micrometric polycrystalline grains, separated by nanoparticle (NP) rich interstices. The nanoparticles act as impurities and promote the formation of a polycrystal, rather than a monocrystal in the absence of added impurities, with well-defined grain boundaries. This wellknown property in solid state systems, specially solid state metallic alloys, is now well documented both in molecular and in distinct colloidal systems [27]. Moreover, the soft methods used to induce micro- and nano-structuring have identified the key solution parameters that influence the microstructure and yield accurate tailoring of it. Such easily functional tuning factors could pave the way for potentially numerous explorations of fundamental mechanisms, such as those mentioned above and reported for solid state atomic systems, particularly the mechanical properties. Indeed, in the 1940s, the pioneering work of Bragg et al. showed that millimetric bubbles floating on water surface interact according to a potential similar to the one encountered in close-packed metals $[35,36]$. As such, they have the paramount advantage of allowing direct visualization of dislocations, grain boundaries and surface deformation around defects. Since then, different types of soft and hard colloids, as well as numerical calculations, have enabled quantitative studies such as nucleation of dislocations and their motion upon contact loading [37]. Although these colloidal analogues to metals have been used extensively, to the best of our 
knowledge, the Hall-Petch relationship has never been reported so far for such a system, whose characteristic length lies in the micrometric level.

The purpose of this study is to explore the evolution of the mechanical properties in a soft colloidal polycrystal. The used system is designed so that the size of the polycrystalline grains is advantageously tuned on a wide micrometric length range. We will explore how the general agreement mentioned above in metallic systems at the nanometric level evolves in a soft colloidal polycrystal. Specifically, how the decrease and increase of the micrometric polycrystalline grain size trigger changes in the rheological properties.

To this end, we produce aqueous solutions of $(\mathrm{EO})_{100-}(\mathrm{PO})_{65}-(\mathrm{EO})_{100}$ copolymer micelles (i.e. polyethylene oxide-polypropylene oxide-polyethylene oxide) to which we add silica NPs whose size (i.e. $\mathrm{D} \approx 26 \mathrm{~nm}$ ) is larger than the micellar diameter (i.e. $\mathrm{D} \approx 20 \mathrm{~nm}$ ). The chosen system is a structurally homogeneous, fluidlike, free-flowing solution below room temperature. Upon heating above the fluid-crystal transition temperature (i.e. $\mathrm{T} \approx 29{ }^{\circ} \mathrm{C}$ ), the micellar solution forms polycrystalline grains in the presence of NPs. We study the mechanical behavior of this soft system by shear rheology and correlate the changes in mechanical parameters to the evolution of the nanostructure and microstructure: the phase transformation towards polycrystalline fcc phase and the size of the polycrystalline grains, monitored by small angle X-ray scattering (SAXS) and phase contrast optical microscopy respectively. We experimentally find that the grain size evolves nonmonotonically with the nanoparticle concentration in this system. Remarkably, our data show that the yield stress rigorously obeys Hall-Petch law.

\section{Materials and methods.}

Materials. The triblock $(\mathrm{EO})_{100}-(\mathrm{PO})_{65}-(\mathrm{EO})_{100}$ copolymer, with a nominal weight average molar mass of $12600 \mathrm{~g} \mathrm{~mol}^{-1}$, was purchased from Sigma-Aldrich. Silica NPs, with an average diameter of $26 \mathrm{~nm}$, were a gift from Nissan Chemical Industries Ltd., Japan. The copolymer was first solubilized in deionized cold water. Then, NPs were added and the samples were stirred and left to rest at $4{ }^{\circ} \mathrm{C}$ for a week. The spherical silica NPs have a very low adsorption rate towards the copolymer which was measured by total organic carbon (TOC) analysis. The data presented here were all taken at a fixed copolymer micellar volume fraction of 15.2 vol. \% (copolymer and silica NP mass densities: 1.05 and $2.4 \mathrm{~g} \mathrm{~cm}^{-3}$ respectively). The samples were investigated at two heating 
rates of 0.1 and $1{ }^{\circ} \mathrm{C} / \mathrm{min}$ from the initial temperature of $10{ }^{\circ} \mathrm{C}$ and up to $42{ }^{\circ} \mathrm{C}$. The heating rates were chosen in order to ensure that evaporation of water is minimal during rheology experiments. The measuring temperature of $42{ }^{\circ} \mathrm{C}$ was chosen because it corresponds to the plateau viscoelastic modulus.

Optical microscopy. The samples were placed in sealed optical cells of $1 \mathrm{~mm}$ thickness and heated to $42{ }^{\circ} \mathrm{C}$ (heating rate $=0.1{ }^{\circ} \mathrm{C} / \mathrm{min}$ ). Several images were recorded during this process. For a given NP concentration, the grains start to form at the onset of fluid-crystal transition temperature and the equilibrium size is very rapidly reached as it is shown in supplementary information on the images in figure 1-SI, taken near the transition temperature point between 29 and $31{ }^{\circ} \mathrm{C}$. For a given NP concentration, the number of polycrystalline grains first increases and rapidly stabilizes when the transition point is exceeded (figure 2-SI in supplementary information). The average size does not evolve with temperature or with time above $29{ }^{\circ} \mathrm{C}$. Once the temperature of $42{ }^{\circ} \mathrm{C}$, at which the rheology measurements were performed, was reached, there was no noticeable texture evolution with time in the course of 3 hours.

At the measuring temperature of $42^{\circ} \mathrm{C}$, several images were taken in different sample locations. The average area of the micellar micrograins was then estimated by dividing the known total surface of the image by the counted number of grains per image. The average diameter of the micrograins was taken as the square root of $4 \times$ grain area/ $\pi$. This average size is probably overestimated for large grains because each image is a random slicing of the samples, as it was noted in reference 28 .

Small angle X-ray scattering (SAXS) measurements were performed on the Swing beamline at synchrotron Soleil (France) at a photon energy of $12 \mathrm{keV}$ and wavelength of $1.03 \AA$. The sampleto-2d detector distance is set to $3.5 \mathrm{~m}$ to cover a wavevector range between 0.0016 and $0.23 \AA^{-1}$. The scattering patterns, which were isotropic, were radially averaged to obtain the scattering intensity as a function of wavevector. The absolute scale $\left(\mathrm{cm}^{-1}\right)$ was obtained by normalization with respect to water at high q. The samples were placed in $1.5 \mathrm{~mm}$ sealed quartz cells and investigated at different temperature points at a heating rate of $0.1^{\circ} \mathrm{C} / \mathrm{min}$. Due to a large difference of the contrast factor between the organic micelles (low contrast factor) and the silica nanoparticles (large contrast factor), the contribution of the nanoparticles to the scattering signal in the polycrystalline phase was straightforwardly obtained by subtracting the scattering signal at $25{ }^{\circ} \mathrm{C}$ 
to the one at $42{ }^{\circ} \mathrm{C}$. Indeed, the contrast between silica NPs and $\mathrm{H}_{2} \mathrm{O}$ solvent, $\left(\rho_{S i-} \rho_{\mathrm{H} 2 \mathrm{O}}\right)^{2}=1.3 \times 10^{-}$ $10 \AA^{-4}$ while for the copolymer, $\left(\rho_{\text {copolymer }-} \rho_{\mathrm{H} 2 \mathrm{O}}\right)^{2}=0.001 \times 10^{-10} \AA^{-4}$. Silica, copolymer and $\mathrm{H}_{2} \mathrm{O}$ scattering length densities $\rho_{S i}, \rho_{\text {copolymer }}$ and $\rho_{H 2 O}$, are $2.080 \times 10^{-5} \AA^{-2}, 0.975 \times 10^{-5} \AA^{-2}$ and $0.943 \times 10^{-5} \AA^{-2}$ respectively. It is thus justified because the micellar signal is at least 2 orders of magnitude weaker than the signal of the nanoparticles, particularly in the wavevector range, around $0.01 \AA^{-1}$, where the NP signal is observed.

Rheology. The measurements of the variation of elastic modulus, G', and viscous modulus, G', as a function of temperature were carried out at a fixed shear frequency of $1 \mathrm{~Hz}$, an applied strain of $1 \%$, and heating rate of $1^{\circ} \mathrm{C} / \mathrm{min}$. They were performed with a Physica RheoCompass MCR 302 rheometer using a cone and plate geometry (diameter $=50 \mathrm{~mm}$, cone angle $=1^{\circ}$ ) fitted with a solvent trap to minimize evaporation. We also checked that $\mathrm{G}^{\prime}$ and $\mathrm{G}$ ' evolution with temperature do not show noticeable difference at the two heating rates of 0.1 and $1{ }^{\circ} \mathrm{C} / \mathrm{min}$, which is in agreement with data reported previously $[28,38]$. Overall, both $G^{\prime}$ and $G$ ”' sharply increase, and show the expected crossover from viscous liquidlike behavior, defined by a weak G" and G' < G", to viscoelastic solidlike behavior. The viscoelastic modulus increases within a very narrow temperature range around the transition temperature of $29^{\circ} \mathrm{C}$.

Shear-rate controlled experiments were carried out using a ribbed parallel plate geometry (diameter $=25 \mathrm{~mm}$ ), fitted with a solvent trap, in order to determine the yield stress. The samples were first placed in the rheometer by choosing either the heating rate of 0.1 or $1{ }^{\circ} \mathrm{C} / \mathrm{min}$. Then, the flow curves were acquired by increasing the shear rate from 0.01 up to $500 \mathrm{~s}^{-1}$. A waiting time of $25 \mathrm{~s}$ was introduced between two successive shear rate points. The data, which consist of shear stress variation as a function of shear rate, were fitted to Herschel-Bulkley model which accurately provides the yield stress. This experimental procedure rigorously follows the protocol described by Jalal et al. in their study of flow behavior of this micellar system [39].

\section{Results and discussion}

Typical optical microscopy images of samples at a fixed copolymer concentration involved in micellization of 15.2 vol. \%, and with increasingly added silica NPs from 0 to 2.92 vol. \% are shown in figure $1(\mathrm{a}-\mathrm{h})$. These images were recorded at a temperature of $42{ }^{\circ} \mathrm{C}$, well above the fluid-crystal transition temperature of $29^{\circ} \mathrm{C}$ for this fixed micellar volume fraction. On this figure we observe that pure micellar solutions (figure 1a) do not show any noticeable difference in the 
microstructure below and above $29^{\circ} \mathrm{C}$. In contrast, samples with added NPs show, upon heating, the appearance of micrograins. They start to form below the onset of fluid to crystal phase transition temperature and occupy the whole sample slightly above it. On the images of figure 1 we notice that the number of contrasted micrograins per unit surface area increases as NP concentration increases from 0 to 1.67 vol. \% while it decreases when the NP concentration increases from 1.67 to 2.92 vol. \%. The variation of the average grain size, $d$, with NP concentration is also shown in this figure (1i) along with the standard deviation of the size distributions (error bars).

This figure (1i) shows two distinct regimes: a decrease in grain size from $100 \mu \mathrm{m}$ to less than 10 $\mu \mathrm{m}$ with increasing NP concentration from 0 to $1.67 \mathrm{vol} . \%$ followed by an increase when the NP concentration is above $1.67 \mathrm{vol} . \%$. The decreasing branch is in agreement with previous studies on comparable micellar systems and different types of particles [26, 28, 29].

In contrast, the observed increasing branch in figure (1i) is an original result, which, to our knowledge such a non-monotonic evolution of grain size has never been observed before in colloidal polycrystals. However, one should bear in mind that second stage size increase is known to experimentally occur during grain refinement of metal alloys such as Al-Si based casting alloys [40]. In this case a fairly well accepted mechanism invokes an unfavorable interaction between the silicon solute and the grain refining molecules (i.e. $\mathrm{TiB}_{2}$ and $\mathrm{TiAl}_{3}$ ) that makes them less effective nuclei for aluminum. In particular, it was proposed that the formation of a $\mathrm{Ti}_{5} \mathrm{Si}_{3}$ intermetallic compound could be the cause of the phenomenon by forming preferentially on the $\mathrm{TiAl}_{3}$ surface due to a good crystallographic lattice matching. Although it could be interesting to push further the analogy on this point between our colloidal system and metal alloys, we did not do so in this work dedicated to the relation between mechanical strength and grain size in a soft colloidal polycrystal. As we will show in the following, grains of a given size appear to contribute in the same way to the mechanical strength whether the sample concentration is below or above the threshold of approximately 1.67 vol. $\%$ and corresponding to the observed minimum size.

The evolution of the local structure of the system was studied as a function of temperature and particle concentration by SAXS. Briefly, the data recorded at $25{ }^{\circ} \mathrm{C}$ (figure 4 of supporting information), on the sample with the micellar concentration $=15.2$ vol. $\%$ and NP concentration $=$ 0 vol. \% show the expected fluidlike scattering intensity on the micellar copolymer solution. As the NPs are added to the micellar solution, the scattering intensity rapidly increases and displays 
the features of solutions of dilute NP dispersions. The large scattering intensity increase is due to the high scattering length density of the silica NPs. As a precaution, the SAXS data at $25^{\circ} \mathrm{C}$ were modelled by using iterative least squares procedure methods to minimize $\chi^{2}$, by considering that only the nanoparticles contribute to the scattering intensity in this two-component system. The resulting fits which account for NP size polydispersity using a log-normal distribution yields a mean NP diameter of $26 \mathrm{~nm}$ and a standard deviation of 0.1 .

The SAXS data recorded at $42{ }^{\circ} \mathrm{C}$ are presented in figure $2 \mathrm{a}$ and show the evolution of the scattering intensities on the pure micellar solution at 15.2 vol. \%, and as a function of increasing NP concentration. First, SAXS data on the pure micellar solution show the expected feature of a fcc crystal according to the succession of the correlation peaks at q values in the ratio of $1: \sqrt{ } 4 / 3: \sqrt{ } 8 / 3$ : $\sqrt{11 / 3}$ : $\sqrt{12} / 3$. Second, the scattering intensity of the samples with NPs shows the dominance of the NP signal in line with the case seen at $25^{\circ} \mathrm{C}$. We also observe that the fcc structural peaks due to the micellar phase are clearly visible in the scattering intensities on samples with low NP concentrations when their scattering contrast is not yet dominant. The successive correlation peaks located at the same q values and in the ratio of $1: \sqrt{ } 4 / 3: \sqrt{ } 8 / 3: \sqrt{ } 11 / 3: \sqrt{ } 12 / 3$ obviously show that the micellar fcc lattice parameter, which is $320 \AA$, is not impacted by the presence of nanoparticles.

The overall shape of the SAXS curves recorded in the presence of particles is close to the NP form factor. However, a close inspection reveals that NP addition triggers a shoulder peak around $\mathrm{q} \sim$ $0.01 \AA^{-1}$. To better quantify this contribution, we subtracted the signal recorded at $25{ }^{\circ} \mathrm{C}$ where the NPs are shown to be homogeneously distributed in the micellar solution from the signal at $42{ }^{\circ} \mathrm{C}$ for each sample. The result yields the scattering signal of NPs affected by the phase transition and show a correlation peak whose amplitude increases as the NP concentration increases and whose position does not vary.

Such evolution suggests that the NPs evolve from homogenously dispersed in the fluidlike micellar phase to locally confined objects in the polycrystalline micellar phase when the temperature increases from room temperature to $42^{\circ} \mathrm{C}$. Overall, the local concentration corresponding to the NP extra peak displayed in figure $2 b$ is estimated $\sim 6.2$ vol. $\%$. We recall that these observations are in agreement with those reported in a comparable micellar system doped with NPs $[38,41]$ and in this same micellar system doped with laponite nanoparticles [26]. We emphasize that in the previous cited studies $[38,41]$, data had shown that not all the NPs are expelled from the crystalline 
micellar grains. However, in the present system, we believe that the majority of the NPs are segregated in the space between the polycrystalline grains, given the fact that the crystal lattice parameters of the micellar network is not affected when $26 \mathrm{~nm}$ NPs are present in the medium (figure 2a).

We then went forward and measured the evolution of the rheological properties as a function of temperature and NP concentration. First, we show in figure 3, the variation of the elastic modulus, $\mathrm{G}^{\prime}$, of the mixtures as a function of temperature and NP concentration. We notice on this figure that when the temperature increases above $27^{\circ} \mathrm{C}$, G' sharply increases, and shows the expected crossover from viscous liquidlike behavior, defined by a weaker $G^{\prime}$ modulus than the viscous modulus, to elastic solidlike behavior [42]. The elastic modulus increases within a very narrow temperature window centered on the transition temperature point of $29{ }^{\circ} \mathrm{C}$. Remarkably, upon addition of NPs, G' curves show two important features. First, the transition temperature does not shift, which points to a fixed copolymer volume fraction involved in micellization in all solutions. Second, the increase of NP concentration indeed leads to an increase of the plateau elastic modulus up to a concentration of $1.67 \mathrm{vol}$. \% followed by a slight decrease above this NP concentration as it is highlighted in the inset of figure 3.

The flow behavior of the pure micellar solution and with added NPs are shown in figure 4. These typical shear-rate controlled flow measurements on polycrystalline phases at $42{ }^{\circ} \mathrm{C}$ and $\mathrm{NP}$ concentration ranging from 0 to $2.92 \mathrm{vol} . \%$ at the heating rate of $0.1{ }^{\circ} \mathrm{C} / \mathrm{min}$ are represented along with Herschel-Bulkley model fits. To recall, all the samples at temperatures below $29{ }^{\circ} \mathrm{C}$ are freeflowing solutions. The data exhibit the features characteristic of yield stress fluids since they only flow when the applied shear stress exceeds the threshold value called yield stress. The evolution of flow curves is easily described by the relation: $\boldsymbol{\sigma}=\boldsymbol{\sigma}_{\boldsymbol{y}}+\boldsymbol{k} \dot{\boldsymbol{\gamma}}^{\boldsymbol{n}}$, known as Herschel-Bulkley model, where $\sigma$ is the shear stress, $\dot{\gamma}$ the shear rate, $\sigma_{y}$ the yield stress, $\mathrm{k}$ the consistency index and $\mathrm{n}$ the flow index. The fit results are also shown in figure 4 as solid curves. A summary of the fitting parameters is given in Table 1 and additional flow measurements are presented in figure 6-SI, in supplementary information. Briefly, we first notice that $\mathrm{n}$ is always very close to 0.37 for all the samples studied and decided to keep it fixed to 0.37 , and that $k$ displays a decreasing average value at the highest NP concentration and thus kept as a variable fitting parameter along with the yield stress. These results are in agreement with data recently reported in the literature for this micellar system [39]. Indeed, it was shown that the flow index lies between 0.34 and 0.46 and the 
consistency index lies between 3 and $16\left(\mathrm{~Pa} \mathrm{~s}^{\mathrm{n}}\right)$ for micellar concentrations around the one studied here.

Thorough investigation of the yield stress at this fixed micellar volume fraction as a function of NP volume fraction was also achieved at $42{ }^{\circ} \mathrm{C}$ and the heating rate of $1{ }^{\circ} \mathrm{C} / \mathrm{min}$. The deduced values of the yield stress at the two heating rates of 0.1 and $1{ }^{\circ} \mathrm{C} / \mathrm{min}$ are shown in figure 5 . The plot of average yield stress at $0.1^{\circ} \mathrm{C} / \mathrm{min}$ is chosen so that to have rational data treatment at this heating rate. The display of all data at $1{ }^{\circ} \mathrm{C} / \mathrm{min}$ shows how the yield stress varies from one flow experiment to another. First, we observe that both data superimpose in the entire investigated NP concentration range. Second, the data display an almost linear increase of the yield stress as a function of addition of NPs into the micellar crystal when the NP concentration increases from 0 to 1.67 vol. \% followed by a decrease above this concentration threshold. Remarkably, the yield stress reaches its maximum value for the sample concentration which displays the shortest grain size. By combining both data of figure $1 \mathrm{i}$ and $5 \mathrm{a}$, the yield stress results are plotted in figure $5 \mathrm{~b}$ and show its variation as a function of the inverse square root of grain size, $\mathrm{d}^{-1 / 2}$. The variation displays a linear increase of yield stress as a function of $\mathrm{d}^{-1 / 2}$ for all the studied samples including concentrated samples above 1.67 vol. \% for which the yield stress values are also distributed around the straight line. It is thus straightforwardly inferred that in finely grained materials, the prediction of Hall and Petch for the variation of yield stress with grain size is fully verified including in soft colloidal polycrystalline samples. The yield stress follows in that the variation of the grain size which is found to be nonmonotonous in this system. The decrease in grain size prompts an increase in yield stress while its increase triggers a decrease of it. A straightforward comparison of data in figure $5 \mathrm{~b}$ with those previously in solid state metallic systems clearly shows that Hall-Petch relation validity extends to soft colloidal systems which involve considerably large length scales including NP impurities and crystal grain sizes.

\section{Conclusion}

As a conclusion, we studied the mechanical strength enhancement by grain size reduction in a soft colloidal polycrystal composed of copolymer micellar crystallites and silica nanoparticles. In this system the fluid-polycrystal phase transition is induced by the variation of temperature and the average size of the grains is tuned by varying the amount of added nanoparticles. The primary result of this study is that the grain size decrease, or grain refinement, induces strength 
improvement. We show a strict linear variation of the yield stress as a function of the inverse square root of grain diameter as predicted by the Hall and Petch relationship. This equation is a major constitutive law governing mechanical behavior in response to grain size variations in atomic materials where the grain size usually lies in the nanometric range. Our system, while it is structurally comparable to a solid state polycrystal, has characteristic lengths which lie in the micrometric level. The second result of this study is that the soft colloidal system we use also shows a non-monotonous grain size variation as a function of added nanoparticle impurities in the grain boundaries. This feature bears resemblance to a similar effect observed in solid state metallic alloys which occurs above an impurity concentration threshold. Our approach could offer the possibility to study new aspects of polycrystalline and nanocrystalline structures and their mechanics with non-destructive experimental methods. They include important issues frequently reported in structural solid state materials such as super-plasticity or the inverse Hall-Petch phenomenon, whose origins remain poorly understood.

\section{Supplementary information}

Description of data analysis, additional results and references are provided.

\section{Acknowledgements}

We thank Dr Thomas Bizien (Soleil synchrotron, Swing beamline) for his assistance with carrying out SAXS experiments. Soleil synchrotron is gratefully acknowledged for beam time allocation. We thank Dr Nicolas Sanson (ESPCI) for his help with TOC measurements. We thank Dr. Cyprien Gay and Dr. Laurent Limat for helpful discussions. 


\section{References}

[1] C. S. Pande, K. P. Cooper, Nanomechanics of Hall-Petch relationship in nanocrystalline materials, Prog. Mater. Sci. 2009, 54, 689-706.

[2] L. Zhong, F. Sansoz, Y. He, C. Wang, Z. Zhang, S. X. Mao, Slip-activated surface creep with room-temperature super-elongation in metallic nanocrystals, Nat. Mater. 2017, 16, 439-445.

[3] Z. Chen, H. Kang, G. Fan, J. Li, Y. Lu, J. Jie, Y. Zhang, T. Li, X. Jian, T. Wang, Grain refinement of hypoeutectic Al-Si alloys with B, Acta Mater. 2016, 120, 168-178.

[4] I. A. Ovid'Ko, R. Z. Valiev, Y. T. Zhu, Review on superior strength and enhanced ductility of metallic Nanomaterials, Prog. Mater. Sci. 2018, 94, 462-540.

[5] P. Y. Huang, C. S. Ruiz-Vargas, A. M. Van Der Zande, W. S. Whitney, M. P. Levendorf, J. W. Kevek, S. Garg, J. S. Alden, C. J. Hustedt, Y. Zhu, J. Park, Grains and grain boundaries in singlelayer graphene atomic patchwork quilts, Nature 2011, 469, 389-392.

[6] F. A. Lavergne, A. Curran, D. G. Aarts, R. P.Dullens, Dislocation-controlled formation and kinetics of grain boundary loops in two-dimensional crystals, Proc. Nat. Acad. Sci. 2018, 115, 6922-6927.

[7] R. A. Masumura, P. M. Hazzledine, C. S. Pande, Yield stress of fine grained materials., Acta Mater. 1998, 46, 4527-4534.

[8] Y. Li, A. J. Bushby, D. J. Dunstan, The Hall-Petch effect as a manifestation of the general size effect, Proc. Roy. Soc. A 2016, 472, 20150890.

[9] L. Y. Chen, M. R. He, J. Shin, G. Richter, D. S. Gianola, Measuring surface dislocation nucleation in defect-scarce nanostructures, Nat. Mater. 2015, 14, 707-713.

[10] L. B. He, L. Zhang, L. P. Tang, J. Sun, Q. B. Zhang, L.T. Sun, Novel behaviors/properties of nanometals induced by surface effects, Mater. Today Nano 2018, 1, 8-21.

[11] I. A. Ovid'Ko, Deformation of Nanostructures, Science 2002, 295, 2386-2386.

[12] S. N. Naik, S. M. Walley, The Hall-Petch and inverse Hall-Petch relations and the hardness of nanocrystalline metals, J. Mater. Sci. 2020, 55, 2661-2681.

[13] S. Wang, Z. Shan, H. Huang, The mechanical properties of nanowires, Adv. Sci. 2017, 4, 1600332 .

[14] Y. Wang, M. Chen, F. Zhou, E. Ma, High tensile ductility in a nanostructured metal, Nature 2002, 419, 912-915. 
[15] M. Murayama, J. M. Howe, H. Hidaka, S. Takaki, Atomic-level observation of disclination dipoles in mechanically milled, nanocrystalline Fe, Science 2002, 295, 2433-2435.

[16] J. Schiøtz, F. D. Di Tolla, K. W. Jacobsen, Softening of nanocrystalline metals at very small grain sizes, Nature 1998, 391, 561-563.

[17] J. Sun, L. He, Y. C. Lo, T. Xu, H. Bi, L. Sun, Z. Zhang, S. X. Mao, J. Li, Liquid-like pseudoelasticity of sub-10-nm crystalline silver particles, Nat. Mater. 2014, 13, 1007-1012.

[18] G. Richter, K. Hillerich, D. S. Gianola, R. Monig, O. Kraft, C. A. Volkert, Ultrahigh strength single crystalline nanowhiskers grown by physical vapor deposition, Nano Lett. 2009, 9, 30483052 .

[19] Y. Yue, P. Liu, Z. Zhang, X. Han, E. Ma, Approaching the theoretical elastic strain limit in copper nanowires, Nano letters 2011, 11, 3151-3155.

[20] P. Pieranski, Colloidal crystals, Contemp. Phys. 1983, 24, 25-73.

[21] A. P. Gast, W. B. Russel, Simple ordering in complex fluids, Phys. Today 1998, 51, 24-31.

[22] B. Senyuk, Q. Liu, S. He, R. D. Kamien, R. B. Kusner, T. C. Lubensky, I. I. Smalyukh, Topological colloids, Nature 2013, 493, 200-205.

[23] W. T. Irvine, A. D. Hollingsworth, D. G. Grier, P. M. Chaikin, Dislocation reactions, grain boundaries, and irreversibility in two-dimensional lattices using topological tweezers, Proc. Nat. Acad. Sci. 2013, 110, 15544-15548.

[24] W. Poon, Colloids as big atoms, Science 2004, 304, 830-831.

[25] C. E. Cash, J. Wang, M. M. Martirossyan, B. K. Ludlow, A. E. Baptista, N. M. Brown, E. J. Weissler, J. Abacousnac, S. J. Gerbode, Local melting attracts grain boundaries in colloidal polycrystals, Phys. Rev. Lett. 2018, 120, 018002.

[26] I. Boucenna, M. A. Guedeau-Boudeville, A. Lapp, P. Colinart, A. Proag, L. Royon, A. Mourchid, Temperature directed-assembly of coated-laponite nanoparticles in pluronic micellar solutions, Soft Matter 2013, 9, 170-176.

[27] S. Gokhale, K. H. Nagamanasa, R. Ganapathy, A. K. Sood, Grain growth and grain boundary dynamics in colloidal polycrystals, Soft Matter 2013, 9, 6634-6644.

[28] N. Ghofraniha, E. Tamborini, J. Oberdisse, L. Cipelletti, L. Ramos, Grain refinement and partitioning of impurities in the grain boundaries of a colloidal polycrystal, Soft Matter 2012, 8, 6214-6219. 
[29] A. Louhichi, E. Tamborini, N. Ghofraniha, F. Caton, D. Roux, J. Oberdisse, L. Cipelletti, L. Ramos, Nucleation and growth of micellar polycrystals under time-dependent volume fraction conditions, Phys. Rev. E 2013, 87, 032306

[30] S. Wu, L. Li, H. Xue, K. Liu, Q. Fan, G. Bai, J. Wang, Size controllable, transparent, and flexible 2D silver meshes using recrystallized ice crystals as templates, ACS Nano 2017, 11, 9898 9905.

[31] X. Shen, L. Chen, D. Li, L. Zhu, H. Wang, C. Liu, Y. Wang, Q. Xiong, H. Chen, Assembly of colloidal nanoparticles directed by the microstructures of polycrystalline ice, ACS Nano 2011, 5, 8426-8433.

[32] S. Hu, J. Nozawa, H. Koizumi, K. Fujiwara, S. Uda, Grain boundary segregation of impurities during polycrystalline colloidal crystallization, Cryst. Growth Des. 2015, 15, 5685-5692.

[33] K. Yoshizawa, T. Okuzono, T. Koga, T. Taniji, J. Yamanaka, Exclusion of impurity particles during grain growth in charged colloidal crystals, Langmuir 2011, 27, 13420-13427.

[34] F. A. Lavergne, S. Diana, D. G. Aarts, R. P. Dullens, Equilibrium grain boundary segregation and clustering of impurities in colloidal polycrystalline monolayers, Langmuir 2016, 32, 1271612724.

[35] W. L. Bragg, J. F. Nye, A dynamical model of a crystal structure, Proc. R. Soc. London 1947, 190, 474-481.

[36] W. L. Bragg, W. M. Lomer, A dynamical model of a crystal structure. II, Proc. R. Soc. London 1949, 196, 171-181.

[37] D. McDermott, C. O. Reichhardt, C. Reichhardt, Avalanches, plasticity, and ordering in colloidal crystals under compression, Phys. Rev. E 2016, 93, 062607.

[38] A. Louhichi, E. Tamborini, J. Oberdisse, L. Cipelletti, L. Ramos, Viscoelasticity of colloidal polycrystals doped with impurities, Phys. Rev. E 2015, 92, 032307.

[39] M. Jalaal,G. Cottrell, N. Balmforth, B. Stoeber, On the rheology of Pluronic F127 aqueous solutions, J. Rheol.2017, 61, 139-146.

[40] D. Qiu, J.A. Taylor, M-X. Zhang, P.M. Kelly, A mechanism for the poisoning effect of silicon on the grain refinement of Al-Si alloys, Acta Materialia 2007, 55, 1447-1456.

[41] E. Tamborini, N. Ghofraniha, J. Oberdisse, L. Cipelletti and L. Ramos, Structure of Nanoparticles Embedded in Micellar Polycrystals, Langmuir 2012, 28, 8562-8570. 
[42] I. Boucenna, L. Royon, M. A. Guedeau-Boudeville, A. Mourchid, Rheology and calorimetry of microtextured colloidal polycrystals with embedded laponite nanoparticles, J. Rheol. 2017, 61, 883-892. 


\section{Figures}
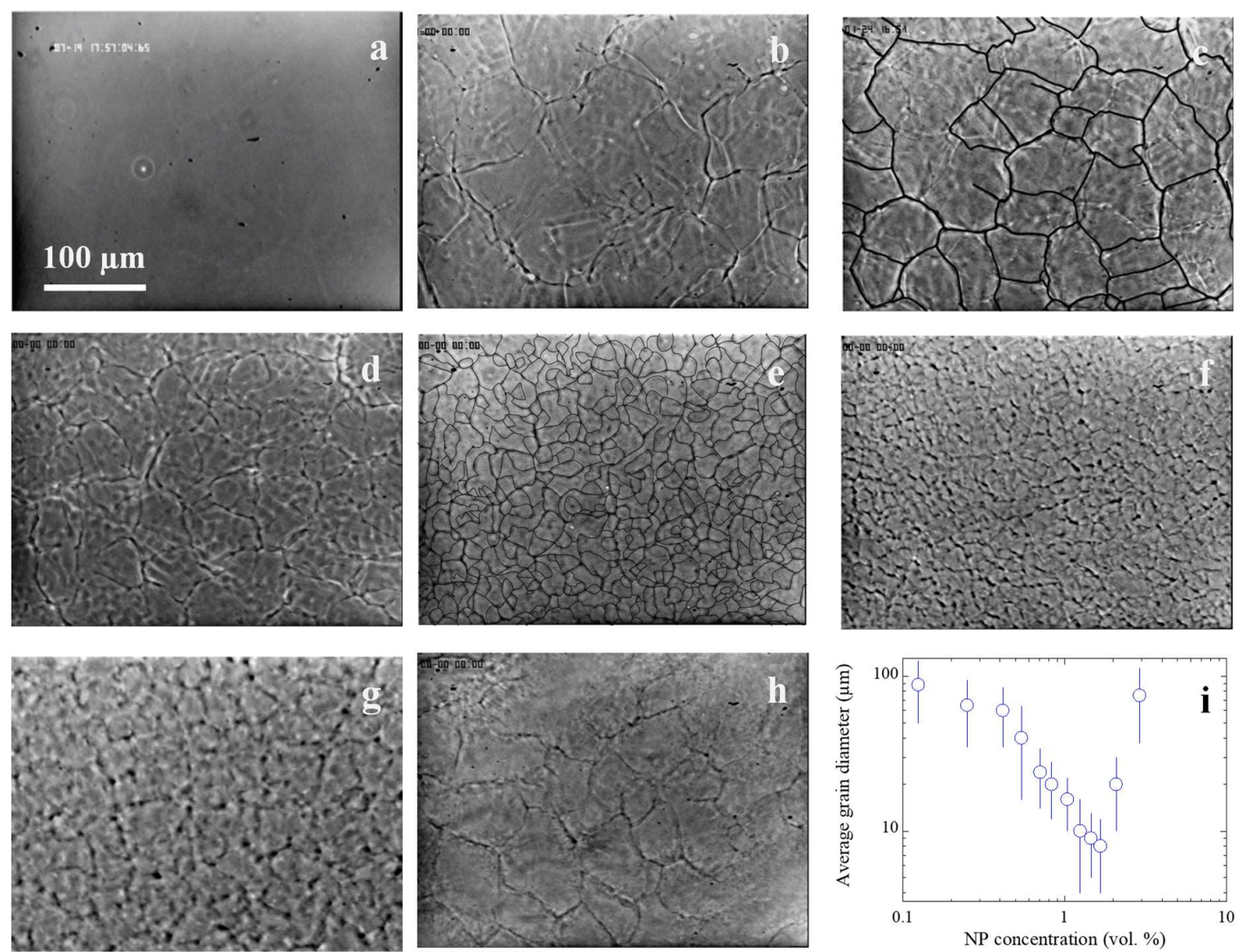

Figure 1. Observation by optical microscopy of samples at a micellar concentration of 15.2 vol. \% and nanoparticle concentration of 0 (a), 0.125 (b), 0.417 (c), 0.833 (d), 1.25 (e), 1.67 (f), 2.08 (g) and $2.92(\mathrm{~h})$ vol. \%. The images were recorded at $\mathrm{T}=42{ }^{\circ} \mathrm{C}$ after a standing time of $15 \mathrm{~min}$. the interfaces in (c) are hand-highlighted. The scale bar is $100 \mu \mathrm{m}$ and holds for all the images. Variation of the average grain size with nanoparticle concentration with error bars (i). 


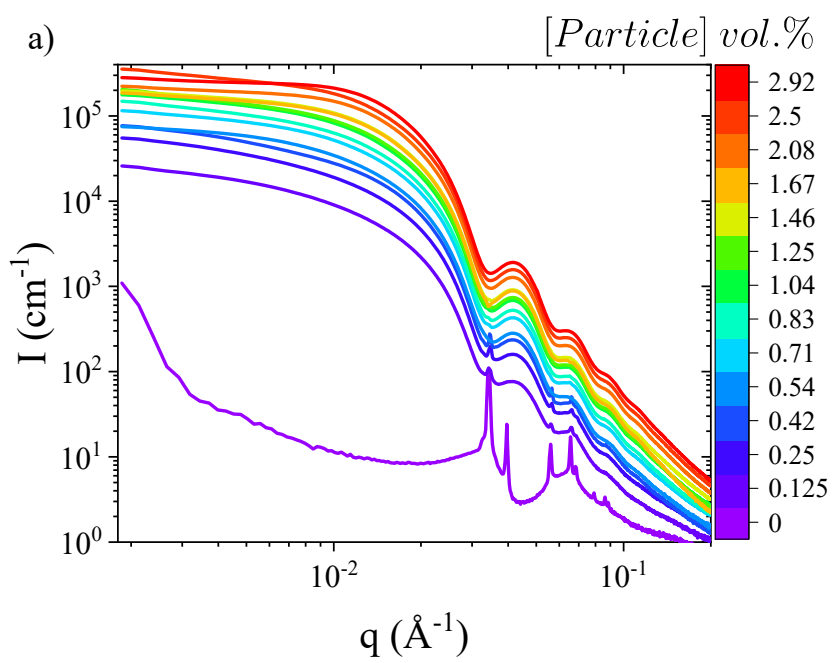

b)

[Particle] vol.\%

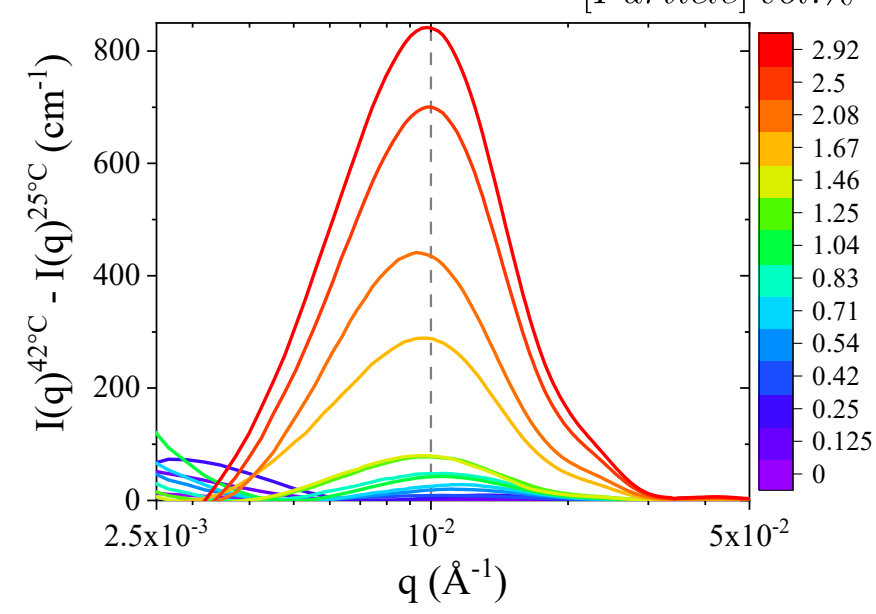

Figure 2. SAXS intensities on samples at a micellar concentration of 15.2 vol. \% and nanoparticle concentration ranging between 0 and 2.92 vol. \%. (a) Representation of $\mathrm{I}(\mathrm{q})$ measured at $\mathrm{T}=42{ }^{\circ} \mathrm{C}$ and (b) Representation of $\mathrm{I}(\mathrm{q})^{42^{\circ} \mathrm{C}}-\mathrm{I}(\mathrm{q})^{25^{\circ} \mathrm{C}}$ in the $\mathrm{q}$ domain where the NP correlation peak appears. 


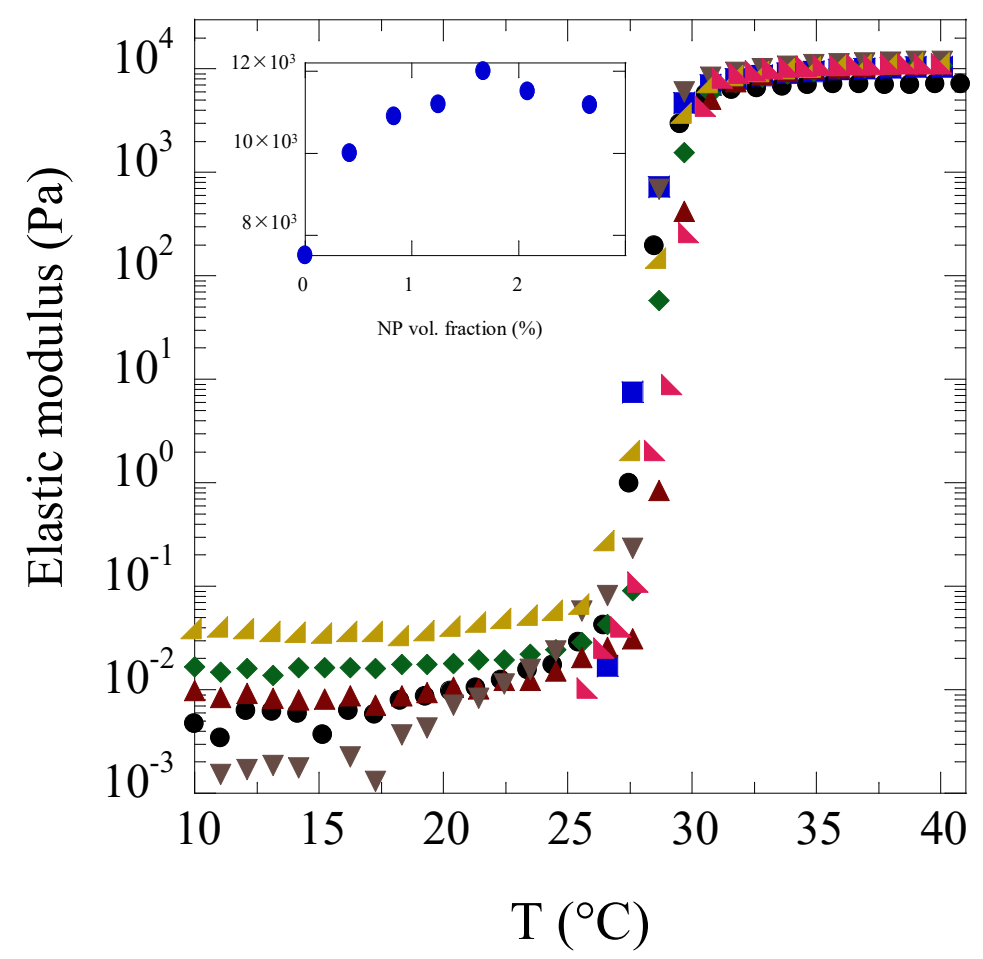

Figure 3. Evolution of elastic modulus as a function of temperature at the micellar concentration of 15.2 vol. $\%$ and nanoparticle concentration of $0,0.42,0.83,1.25,1.67,2.08$ and 2.70 vol. \% (colored $\boldsymbol{\odot}, \boldsymbol{\square}, \boldsymbol{\nabla}, \boldsymbol{\Delta}, \boldsymbol{\nabla}, \boldsymbol{\Lambda}$ and $\mathbf{\Delta}$ respectively). Inset: the variation of plateau elastic modulus as a function of NP concentration. 


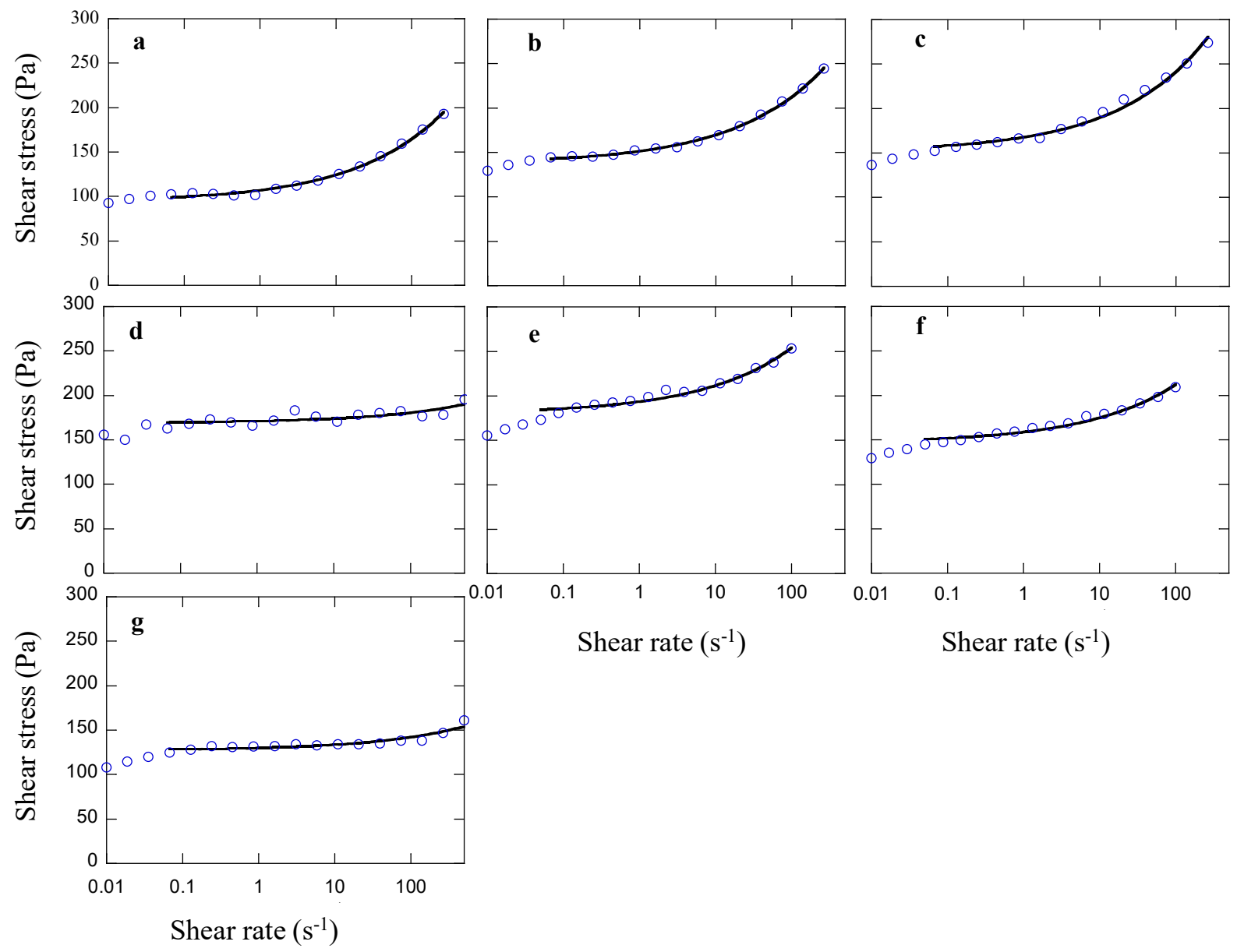

Figure 4. Evolution of flow curves at $42{ }^{\circ} \mathrm{C}$, micellar concentration of 15.2 vol. \%, and nanoparticle concentration of $0,0.42,0.83,1.25,1.67,2.08$ and 2.92 vol. \% (empty circles, a, b, c, d, e, f and g respectively). Fits to Herschel-Bulkley model with corresponding yield stress values of 94, 137, 151, 180, 147, and $127 \mathrm{~Pa}$ (solid lines, a, b, c, d, e, f and g respectively) performed between 0.08 $\mathrm{s}^{-1}$ and maximum shear rate reached. 

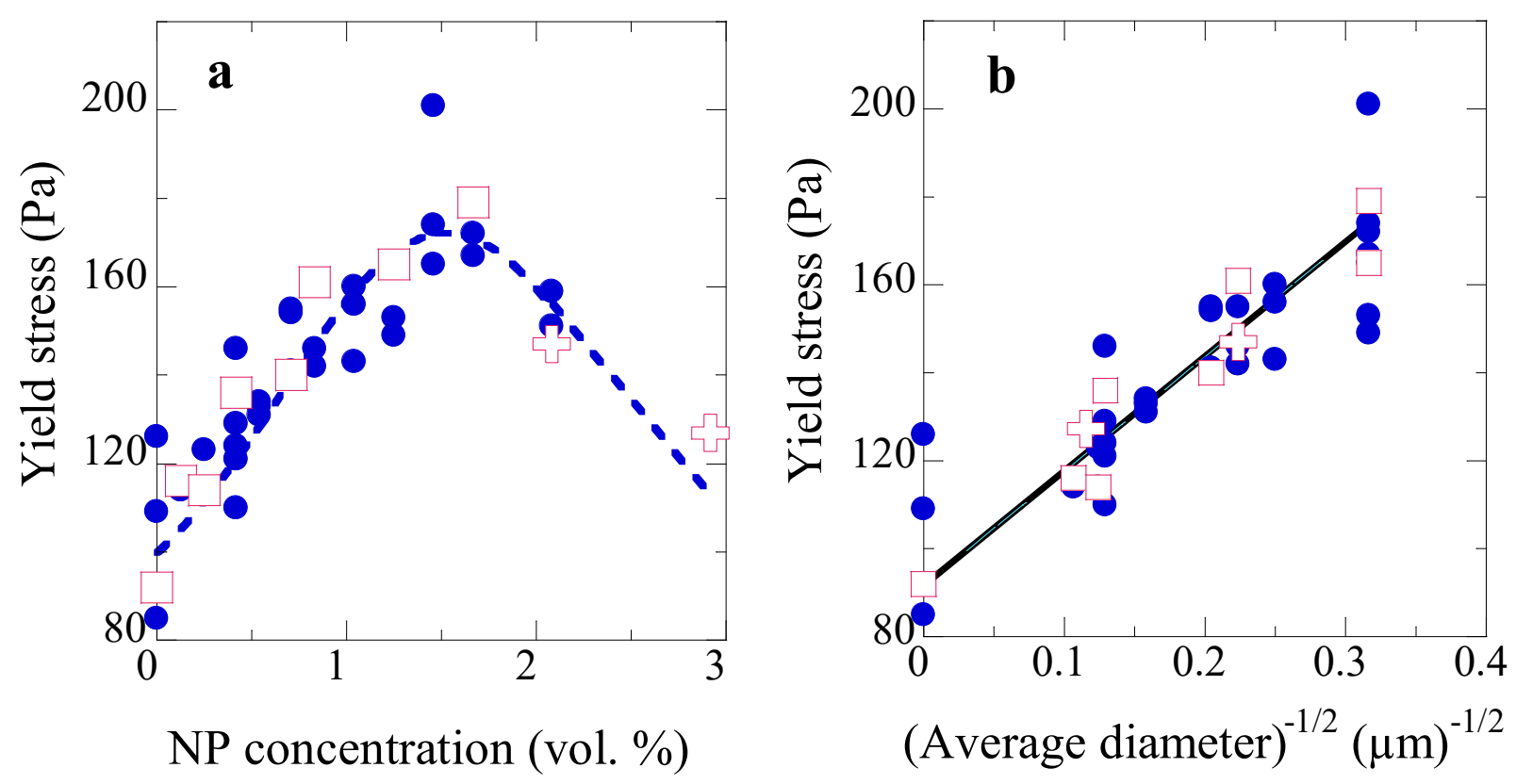

Figure 5. (a) Variation of yield stress as a function of nanoparticle concentration at $42{ }^{\circ} \mathrm{C}$ and heating rate of 1 (full circles) and 0.1 (empty squares) ${ }^{\circ} \mathrm{C} / \mathrm{min}$. Each square and plus symbol point is an average of 3 to 5 experiments; the dashed curve is a guide to the eye. (b) Representation of yield stress recorded at $42{ }^{\circ} \mathrm{C}$ as a function of inverse square root of micellar grain diameter at a heating rate of 1 (full circles) and $0.1{ }^{\circ} \mathrm{C} / \mathrm{min}$ (squares and pluses). The squares correspond to the increasing branch of figure 5a (nanoparticle concentration from 0 to 1.67 vol. \%) while the pluses correspond to its decreasing branch (nanoparticle concentration from 1.67 to 2.92 vol. \%). The solid line is a least squares linear fit at heating rate of $0.1^{\circ} \mathrm{C} / \mathrm{min}$. 